\title{
Plasma endothelin in coronary venous blood from patients with either stable or unstable angina
}

\author{
J T Stewart, J A Nisbet, M J Davies
}

\section{Department of Cardiological Sciences, St George's Hospital Medical School, London J T Stewart \\ Department of Chemical Pathology, St George's Hospital Medical School, London \\ J A Nisbet \\ Department of Cardiovascular Pathology, St George's Hospital Medical School, London M J Davies \\ Correspondence to Dr J T Stewart, Royal Brompton National Heart and Lung Hospital, Sydney Street, London SW3 6NP.}

\begin{abstract}
Study objective-To test the hypothesis that the active coronary endothelial lesions in unstable angina raise the endothelin concentration in coronary venous blood.

Design-Systemic and coronary venous blood samples were obtained from unselected patients with the clinical syndromes of either stable or unstable angina at the time of cardiac catheterisation and coronary arteriography. Control venous blood samples were obtained from healthy laboratory workers and from patients with chronic renal failure treated by intermittent haemodialysis.
\end{abstract}

Patients-Twelve patients with angina: seven with stable symptoms and five with unstable angina.

Results-The mean coronary venous endothelin concentration in unstable angina was $2 \cdot 32 \mathrm{ng} / 1$ (range 1.7-3.2 $\mathrm{ng} / 1$ ). In stable angina it was $2.77 \mathrm{ng} / \mathbf{1}$ (range $2 \cdot 1-4 \cdot 4 \mathrm{ng} / 1)$. These values were not significantly different from one another nor from the values obtained in systemic venous blood from either group or from the healthy controls. Circulating endothelin concentrations were much higher in venous blood from the patients treated by haemodialysis.

Conclusions-These data do not support the hypothesis that raised endothelin concentrations in coronary blood in patients with unstable angina may modulate variations in coronary arterial tone thereby contributing to the clinical syndrome of chest pain and electrocardiographic changes at rest. The raised endothelin concentrations seen in systemic venous blood after myocardial infarction may be part of the systemic response to myocardial infarction.

There is increasing evidence that the vascular endothelium helps to regulate vascular smooth muscle tone. Endothelium dependent vasodilatation was first reported in $1980,{ }^{1}$ and a vasoconstrictor substance derived from cultured endothelial cells, now called endothelin, was reported some years later. ${ }^{2-4}$ Although its exact physiological role is not yet known, endothelin mediated vasoconstriction may be induced by various hormonal, neural, and mechanical stimuli. Endothelin release is stimulated by, among other things, thrombin ${ }^{4}$ and sheer stress, ${ }^{5}$ suggesting that it may play a role in local haemostasis and implying that an important stimulus to its release is local endothelial cell injury. High circulating concentrations of endothelin have also been discovered in patients with acute renal failure ${ }^{6}$ and in those with chronic renal failure receiving haemodialysis, but not in those with chronic renal failure who are not receiving dialysis. ${ }^{7}$ Endothelial injury, particularly during haemodialysis, has been proposed as the explanation for this observation. The reported intraoperative increase in circulating endothelin concentrations in patients undergoing abdominal surgery was attributed to vascular endothelial cell injury during surgical manipulation. ${ }^{8}$

In acute coronary syndromes, including unstable angina, there is endothelial damage and thrombus formation in the coronary circulation. $^{9-11}$ On theoretical grounds such stimuli might be expected to stimulate endothelin production in the coronary circulation. If this were so, it might help to explain the apparently spontaneous variations in coronary tone seen in unstable angina and "stuttering myocardial infarction". Thus endothelin released locally as a result of endothelial injury and thrombus formation might influence symptoms and outcome in acute coronary syndromes.

\section{Patients and methods}

Endothelin concentrations were assayed in coronary sinus blood and in paired samples of systemic venous blood in 12 patients (eight men and four women, aged 40-73) with angina, at the time of cardiac catheterisation and coronary arteriography. Seven patients had chronic stable angina; in five of them $(71 \%)$ there was multivessel coronary disease. The other five had unstable symptoms, with chest pain and ST segment depression at rest, from 4 to 24 hours before catheterisation; multivessel coronary disease was present in three $(60 \%)$. All the patients with unstable angina were receiving intravenous nitrate and heparin infusions at the time of angiography. No patient, in either group, complained of angina during coronary arteriography. Informed consent was obtained before the procedure and all patients had been fasting for at least six hours before catheterisation was performed. The results were compared with endothelin concentrations assayed in samples of venous blood obtained from patients with chronic renal failure treated with regular haemodialysis.

Before angiography took place in 10 
patients the coronary sinus was intubated with a polyethyline $7 F$ Sones catheter (Cordis UK, Brentford, Middlesex), positioned from the left subclavian vein. In the remaining two patients, the coronary sinus was intubated with a woven Dacron thermodilution catheter (Webster Laboratories, Baldwin Park, California) approximately 10 minutes after coronary arteriography had been performed. In all patients the tip of the catheter was positioned approximately $2.5 \mathrm{~cm}$ from the os of the coronary sinus, to minimise the risk of admixture of right atrial blood in the samples; a satisfactory catheter position was confirmed by the hand injection of radiographic contrast.

Paired $8 \mathrm{ml}$ samples of coronary venous and systemic venous (either right atrial or pulmonary arterial) blood were collected into refrigerated tubes containing sodium EDTA $1.5 \mathrm{mg} / \mathrm{ml}$ and aprotonin $500 \mathrm{KIU} / \mathrm{ml}$ and stored on ice. Samples were centrifuged at $2^{\circ} \mathrm{C}$, separated within an hour of collection, and stored at $-70^{\circ} \mathrm{C}$ for up to three months (two months on average). During this period venous blood was also collected from a group of healthy controls and from a group of patients with chronic renal failure, on a programme of regular haemodialysis.

Endothelin was assayed according to the Peninsula Laboratories' protocol, modified to increase sensitivity by extracting $4 \mathrm{ml}$ plasma instead of $2 \mathrm{ml}$. Acidified plasma was applied to a C18 Sep column, and after this was washed with $0.1 \%$ trifluoroacetic acid endothelin-like materials were eluted with $60 \%$ acetonitrile in $0.1 \%$ trifluoroacetic acid. The eluant was evaporated to dryness and the residue was reconstituted in $0.6 \mathrm{ml}$ of buffer. Endothelin was then measured with the Peninsula radioimmunoassay kit. Low concentration and high concentration quality control samples were prepared by adding endothelin to EDTA plasma, stored in aliquots at $-70^{\circ} \mathrm{C}$, and assayed in each batch throughout the study period. Recovery of endothelin throughout the entire procedure was assessed by the addition of $4 \mathrm{ng} / \mathrm{l}$ and $32 \mathrm{ng} / \mathrm{l}$ endothelin to EDTA plasma from healthy controls.

\section{Results}

The mean plasma endothelin concentration in coronary sinus blood in the patients with unstable angina was $2 \cdot 32 \mathrm{ng} / 1$ (range $1 \cdot 7-3 \cdot 2$ ), and in the patients with stable symptoms it was $2 \cdot 77 \mathrm{ng} / 1$ (range $2 \cdot 1-4 \cdot 4 \mathrm{ng} / \mathrm{l}$ ). These values were not significantly different from one another or from the mean values obtained in systemic venous blood-that is, 2.42 (range $1.9-3.5) \mathrm{ng} / 1$ and 2.57 (range $2 \cdot 0-3 \cdot 1$ ) $\mathrm{ng} / \mathrm{l}$ in the unstable and stable groups respectively. The endothelin values in systemic blood from the patient groups were similar to those found in 15 healthy laboratory workers (mean $3.0 \mathrm{ng} / \mathrm{l}$, range $2 \cdot 0-5 \cdot 9)$. In contrast, circulating endothelin concentrations in the patients receiving haemodialysis for chronic renal failure were much higher, with a mean value of $8.4 \mathrm{ng} / 1$ (range $5 \cdot 0-13 \cdot 3 \mathrm{ng} / \mathrm{l}$ ), which accords with that

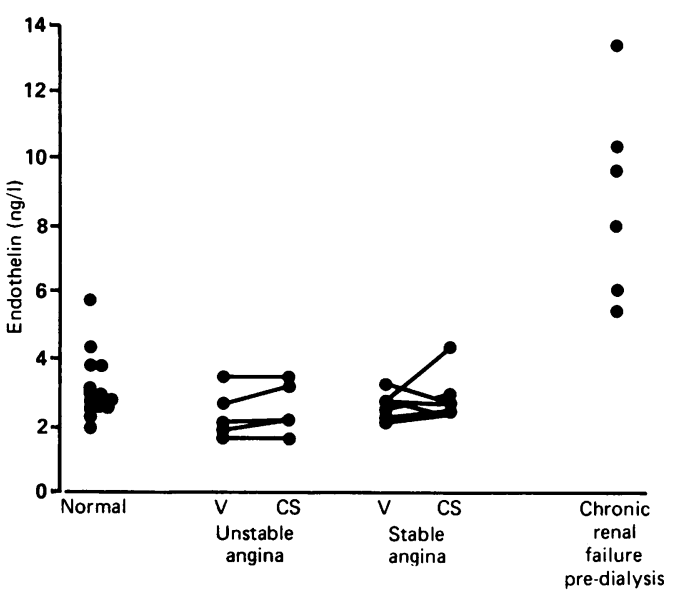

Endothelin concentrations in systemic ( $V$ ) and coronary (CS) venous blood in patients with unstable or stable angina and in systemic venous blood from healthy laboratory workers and from patients with chronic renal failure.

reported in such patients by other groups. ${ }^{7}$ The figure shows the results.

Recovery of added endothelin was $84 \%$ (range $75-89 \%, \mathrm{n}=6$ ) and $98 \%$ (range 76 $118 \%, n=4)$ for the addition of 4 and $32 \mathrm{ng} / 1$ respectively. Endothelin values (mean (SD)) in the low concentration and high concentration quality control samples, analysed with each batch, were $7.85(2 \cdot 18) \mathrm{ng} / \mathrm{l}$ and $38.3(8 \cdot 0) \mathrm{ng} / \mathrm{l}$ respectively $(n=8)$. In the high concentration quality control sample, however, the endothelin values were approximately $30 \%$ lower after 7.5 months storage at $-70^{\circ} \mathrm{C}$.

\section{Discussion}

We did not show an increase in endothelin concentration in the coronary circulation or in the systemic circulation of patients with either stable or unstable angina. The kinetics of endothelin synthesis and release are such that we would have expected to detect raised concentrations in the coronary venous blood of these patients if endothelin-modulated variations in coronary arterial tone contributed to the chest pain and electrocardiographic changes of unstable angina.

These data must be interpreted in the light of recently published work from Yasuda et al, who showed raised plasma endothelin concentrations in systemic venous blood from nine patients with acute myocardial infarction but not from 10 patients with stable angina. ${ }^{12}$ Endothelin concentration in venous blood was highest within 24 hours of myocardial infarction and fell gradually over a period of 14-16 days to the value found in controls who did not have coronary artery disease. In the infarct patients there was a significant association between plasma endothelin concentration and left ventricular wall motion abnormality, and between plasma endothelin concentration and systemic markers of activation of the coagulation system (thrombin-antithrombin III complex, and $\beta$-thromboglobulin), but not between endothelin concentration and systemic blood pressure. The highest concentrations seen, however, were in two patients in Killip subset 
IV-that is in the two patients with the largest infarcts and greatest haemodynamic disturbance.

The source of the endothelin in the infarct patients was not identified, but the inference was that it arose in the coronary circulation. Unlike other vasoactive peptides found in the coronary circulation, endothelin is not stored in subcellular vesicles. This makes it unlikely that it could arise from lysed endothelial cells within the infarct, but stimulated endothelial cells in the border zone of the infarct are a possible source. Another possibility is that the endothelin was produced in extracardiac sites as part of the systemic response to myocardial infarction, perhaps secondary to the hypercoagulability and platelet hyperreactivity seen in acute myocardial infarction or to increased sympathetic activity. (The same group has published data showing that adrenaline increased endothelin production from cultured porcine endothelial cells ${ }^{13}$.)

Yasuda et al speculated on the possible physiological or pathophysiological effects of endothelin in acute myocardial infarction There may be a role for a powerful vasoconstricting and positively inotropic agent in the patient who is hypotensive after infarction, and, despite the lack of a significant association between endothelin concentration and systemic blood pressure in this study, the high concentrations in the two patients in Killip class IV are intriguing. Raised concentrations were found in patients with cardiogenic shock, whereas, in the same study, the concentrations in patients with stable congestive heart failure were not raised. ${ }^{14}$ Our data, which show no increase in endothelin concentration in coronary sinus blood or systemic venous blood from patients with unstable angina pectoris support the contention that the raised endothelin production was part of the systemic response to acute myocardial infarction.

The assay showed the expected high concentrations of circulating endothelin in patients with chronic renal failure on haemodialysis, which suggests that the low concentrations measured in the patients with coronary artery disease were probably valid, although the quality control data showed that assay precision during the study was not good. The high concentration quality control sample seemed to be unstable, which casts doubt on the reliability of the data based on it, at least in the assessment of precision. No decrease in endothelin was seen for the low concentration quality control sample, but this could have been masked by imprecision. If there had been any loss in endothelin activity in the patients' samples it would not have explained our failure to detect a difference in endothelin concentration between venous and coronary sinus blood. Paired samples of systemic and coronary venous blood were stored for the same length of time and were analysed together in the same batch. It is still possible, however, that small increases in endothelin concentration in coronary sinus blood compared with systemic venous blood remained undetected by the methods we used. If this were so, a sensitive immunometric assay without column extraction would have been more appropriate.

Although endothelin is not available for immediate release, its synthesis in the face of endothelial injury seems to be rapid, as is its appearance in systemic blood after myocardial infarction. ${ }^{12}$ We would expect, therefore, that patients with symptoms of unstable angina for several days and active intimal lesions would be producing endothelin. We were not able to show an increase in endothelin concentration in the coronary circulation, and it is still not certain whether endothelin helps to modulate coronary vasomotor tone in unstable angina pectoris. Its origin and its role in patients with acute myocardial infarction remain to be established.

1 Furchgott RF, Zawadzki JV. The obligatory role of endothelial cells in the relaxation of arterial smooth muscle by acetylcholine. Nature 1980;288:373-6.

2 Gillespie MN, Owasoyo JO, McMurtry IF, O'Brien RF. Sustained coronary vasoconstriction provoked by a peptidergic substance released from endothelial cells in culture. J Pharmacol Exp Ther 1986;236:339-43.

3 O'Brien RF, Robbins RJ, McMurtry IF. Endothelial cells in culture produce a vasoconstrictor substance. J Cell Physiol 1987;132:263-70.

4 Yanagisawa M, Kurihara H, Kimura S, et al. A novel potent vasoconstrictor peptide produced by vascular endothelial cells. Nature 1988;332:411-5

5 Yoshizumi M, Kurihara H, Sugiyama T, et al. Hemodynamic shear stress stimulates endothelin production by cultured endothelial cells. Biochem Biophys Res Commun 1989;161:859-64.

6 Tomita K, Ujie K, Nakanishi T, et al. Plasma endothelin levels in patients with acute renal failure. $N \mathrm{Engl} \mathrm{J} \mathrm{Med}$ 1989;321:1127.

7 Koyama H, Nishzawa Y, Morii H, Tabata T, Inoue T, Yamaji $T$. Plasma endothelin levels in patients with uraemia. Lancet 1989;i:991-2.

8 Hirata Y, Itoh K-I, Ando K, Endo M, Marumo F. Plasma endothelin levels during surgery. $N$ Engl J Med 1989;321:1686.

9 Davies MJ, Thomas AC. Plaque fissuring - the cause of acute myocardial infarction, sudden ischaemic death, and crescendo angina. Br Heart J 1985;53:363-73.

10 Falk E. Unstable angina with fatal outcome: dynamic coronary thrombosis leading to infarction and/or sudden eath. Autopsy evidence of recurrent mural thrombus with peripheral embolization culminating in total vascular occlusion. Circulation 1985;71:699-708.

11 Sherman CT, Litvak F, Grundfest W, et al. Coronary angioscopy in patients with unstable angina pectoris. $N$ Engl J Med 1986;315:913-9.

12 Yasuda M, Kohno M, Tahara A, et al. Circulating immunoreactive endothelin in ischemic heart disease. Am Heart $J$ 1990;1 19:801-6.

13 Kohno M, Murakawa K, Yokokawa K, et al. Production of endothelin by cultured porcine endothelial cellsmodulation by adrenaline. J Hypertens 1989; 7(suppl 6):S130-1

14 Cernacek P, Stewart DJ. Immunoreactive endothelin in human plasma: marked elevations in cardiogenic shock. Biochem Biophys Res Commur 1989;161:562-7. 